\title{
MedienPädagogik
}

Zeitschrift für Theorie und Praxis der Medienbildung

Themenheft Nr. 28: Tagungsband: Bildung gemeinsam verändern: Diskussionsbeiträge und Impulse aus Forschung und Praxis. Herausgegeben von David Meinhard, Valentin Dander, Andrea Gumpert, Christoph Rensing, Klaus Rummler und Timo van Treeck.

\section{Der Mehrwert des Medieneinsatzes in der Hochschullehre am Beispiel Studierenden- erstellter Videos}

Regina Stober

\begin{abstract}
Zusammenfassung
In der Vergangenheit wurde die Diskussion um Medien in der Hochschullehre vielfach auf technischer Ebene geführt. Dahinterstehende pädagogische Konzepte sowie didaktische Modelle wurden eher vernachlässigt, was mitunter den Lernprozess, der durch den Medieneinsatz unterstützt werden soll, aus dem Blickfeld rückte. Die voranschreitende Technisierung eröffnet eine Vielfalt an Lernszenarien, was Lehrende vor die Herausforderung stellt, sich im ¿Dschungel〉 an Möglichkeiten zurechtzufinden, um eine sinnvolle, der Lerngruppe angepasste Lernumgebung zu gestalten. Ein Indikator, die Eignung eines Mediums für die eigene Lehre abzuleiten, ist nach dem Mehrwert bezüglich der Lernergebnisse zu fragen. In der wissenschaftlichen Diskussion um den Einsatz audiovisueller Medien in der Lehre wurde seit den 1960er Jahren immer wieder der fehlende Tiefgang eines Medien-gestützten Lernprozesses im Vergleich zum Buch bemängelt. Die Skepsis hält an, während der Medieneinsatz in der Lehre zunimmt. Dass die Nutzung audiovisueller Medien - eingebettet in ein sinnvolles didaktisches Szenario - nachhaltige Lernprozesse anregen kann, wird in diesem Beitrag aufgezeigt. Es wird ein mediengestütztes Lernszenario beschrieben, bei dem Studierende selbst Lehrfilme erstellt haben sowie nach dem Mehrwert des Medieneinsatzes gefragt.
\end{abstract}

The benefit of student created videos in higher education

\begin{abstract}
Historically, discussions about the use of media in higher education has been conducted mainly on a technical level. Pedagogical concepts and the learning process itself have been neglected, which instead should have been assisted by the use of media. The growing use of technology opened a variety of potential learning environments. Lecturers therefore, have found it challenging to find their way through the jungle of options > to mold a reasonable and customized learning setting for their learning groups. One indicator that can help finding a way is to look at the benefit of media use for the students Learning Outcomes. The debate - that started back in the 1960s - about the use of audiovisual media in teaching, criticized the absence of depth in medialized learning processes,
\end{abstract}


as compared to a conventional book. This scepticism continues to exist while the use of media in teaching is increasing. This article illustrates that the use of audio-visual media can initiate deep learning processes, if it would be embedded into a reasonable didactical environment. It describes a learning setting supported through films created by students to argue the benefit of media use in specific situations.

\section{Ausgangslage}

Lernende zeichnet heutzutage aus, dass sie überall auf Lernmaterialien zugreifen und damit Ort und Zeit ihres Lernens flexibel bestimmen können (Mürner und Polexe 2014, 5). Die Technisierung des Alltags ermöglicht alle nötigen Informationen auf den eigenen Endgeräten fast überall parat zu haben und in den privaten Alltag zu integrieren. Doch ohne real wahrnehmbaren Mehrwert und bei knappen Ressourcen stossen mediengestützte Lernszenarien auf wenig Akzeptanz (vgl. Kleimann und Wannemacher 2004). Der Mehrwert wird somit zur Grundlage, um den Einsatz von Medien zu reflektieren und sinnvolle Lernumgebungen zu gestalten. Schliesslich ist Medieneinsatz oft mit Mehraufwand für Lehrende und/oder Studierende verbunden, den alle Beteiligten vermutlich nur dann bereit sind zu tragen, wenn ein signifikanter Mehrgewinn zu erwarten ist. Dass es sich lohnen kann, Medien einzusetzen und welchen Mehrwert Studierenden-erstellte Videos mit sich bringen können, wird in diesem Beitrag anhand eines Praxisbeispiels dargestellt. Argumentiert wird, dass nachhaltige Bildungsprozesse durch mediale Lernangebote angeregt werden, wenn die Bedingungen einer Lernsituation stimmen, was in diesem Fall durch eine hohe Identifikation mit dem Lerngegenstand und einer veränderten Lehrendenrolle zu mehr Motivation und Aktivierung der Studierenden führte. ${ }^{1}$

\section{Praxisbeispiel}

Die Teilnehmenden des Pflichtmoduls «Einführung in die Fachdidaktik Religion» (i. d. R. 20 bis 40 Personen) der Universität Hamburg waren Bachelorstudierende aller Lehramtsformen mit dem Fach evangelische Religion im Sommersemester 2013. Folgende Lernziele wurden, in Abstimmung mit den fachspezifischen Bestimmungen, formuliert:

1 Den Wert eines Mediums bestimmt also erst die konkrete Situation und nicht das Medium selbst (vgl. de Witt und Kerres 2002, 19; Kerres 2013, 128). 
- Die Teilnehmenden benennen religionspädagogische Ansätze in Deutschland seit 1945. Sie können sie zeitlich einordnen, Vertreter und Merkmale beschreiben und die Ansätze kritisch reflektieren. Sie sind sich der besonderen Rolle des Hamburger «RU für alle» bewusst; grenzen inn im Vergleich zu anderen Bundesländern ab.

- Die Teilnehmenden reflektieren Methoden der Veranstaltung in Hinblick auf den Einsatz im Religionsunterricht.

- Die Teilnehmenden erstellen Videos, in denen sie eine selbstgewählte Fragestellung zu den Seminarinhalten bearbeiten und analysieren.

Die Lehrform war, prüfungsordnungsgemäss, in Seminar (3 SWS) und Vorlesung (1 SWS) unterteilt. Dabei gab die Vorlesung eine Übersicht über religionspädagogische Ansätze in Geschichte und Gegenwart, während das Seminar aufbauend inhaltliche und methodische Grundfragen des (Hamburger) Religionsunterrichts thematisierte. Der Vorlesungsanteil wurde in den ersten Semesterwochen in Form frontaler Inputs mit integrierten Gruppenarbeitsphasen abgehalten. Die Studierenden erlangten damit das religionspädagogische Theoriewissen, das sie für die anschliessende Erstellung eigener Videos benötigten. Die Motivation bei den Teilnehmenden war recht gross, sich das neue Wissen anzueignen und über die verschiedenen Ansätze zu diskutieren, da sie diese Inhalte für ihr eigenes Projekt anwenden konnten. Während der Theoriephase wurde bewusst eine methodische Vielfalt geboten (Power Point, Postersession, Gruppenpuzzle, Rollenspiel etc.), die reflektiert wurde, um die Methodenkompetenz der angehenden Lehrpersonen zu erweitern. Zusätzlich verdeutlichte eine Reflexionsphase am Ende jeder Sitzung die Relevanz der Seminarinhalte unter den Fragestellungen: «Was nehme ich für meinen Religionsunterricht aus dem Ansatz mit?» und «Von welchen Aspekten des Ansatzes möchte ich mich und meinen Religionsunterricht bewusst abgrenzen?»

Der Einstieg in die Filmphase erfolgte über eine Einführung in das Filmequipment, das den Studierenden über das Medienzentrum zur Verfügung stand. Zur Veranschaulichung, in welche Richtung die Videos der Studierenden gehen könnten, wurden Lehrfilme, die in anderen Veranstaltungen des Arbeitsbereichs entstanden sind, gezeigt und diskutiert. An dieser Stelle war viel Raum, um medienanalytische Kompetenzen anzubahnen, indem die Filme kritisch auf ihre Inhalte und Wirkung diskutiert wurden. Die Studierenden leiteten anschliessend selbständig in eine angeregte Ideensammlung über. Für die folgenden Termine wurden feste fünfer-Gruppen gebildet, in denen einer eigenen Forschungsfrage nachgegangen wurde. Einige Pflichttermine wurden zur freien Verfügung gestellt. Hier wurden Interviews am Campus geführt, Filmszenen gedreht, begutachtet und geschnitten. In der letzten Sitzung wurden alle Filme gemeinsam angeschaut und besprochen. Bei der Themenfindung und Formulierung der Forschungsfrage halfen das im Vorlesungsteil erworbene Wissen, eine kleine Einführung in das forschende Lernen sowie Tipps zur Formulierung guter 
Forschungsfragen. Eine Gruppe beschäftige sich mit der Frage, wie es überhaupt zum Hamburger Religionsunterricht für alle gekommen sei. Sie entschlossen sich, die Inhalte der vorigen Theoriesitzungen in einem Lege- und Zeichenvideo zu vertiefen. Das Ergebnis kann in Zukunft als Anker-Film für andere Veranstaltungen dienen. Eine andere Gruppe setzte ihren Fokus auf den problemorientierten Religionsunterricht und interviewte dazu den Religionspädagogen Horst Gloy. Diese Beispiele zeichneten sich durch einen intensiven Gruppenprozess und qualitative Ergebnisse aus, die in Zukunft weiterhin genutzt werden können. Im gesamten Prozess der Videoentwicklung arbeiteten die Studierenden zunehmend selbständig. Die Lehrendenrolle kann als Lernbegleitung beschrieben werden, da die Studierenden selbstständig arbeiteten, jedoch jederzeit Unterstützung erbeten konnten, insbesondere im Rahmen regelmässiger Berichterstattungen über ihren Arbeitsstatus.

\section{Wesentliche Erkenntnisse aus der Reflexion der Veranstaltung}

Die neueren Entwicklungen in der Hochschullehre beschreiben Mürner und Polexe (2014) als eine neue Lernkultur, die auch neue Lehrendenrollen mit sich bringt. Die Studierenden akzentuierten im Abschlussfeedback das Arbeiten und Lernen auf Augenhöhe, sowohl untereinander, als auch mit der Lehrenden. Wie im Beispiel der beschriebenen Veranstaltung werden Lehrende zu Begleitenden individueller Lernprozesse, während Lernende immer mehr mit selbstgesteuerten und kooperativen Aufgaben konfrontiert werden, zu deren Bewältigung sie sowohl medien- als auch fachkompetente Betreuung durch die Lehrenden benötigen. Die Beurteilung der Lehre kann auf verschiedene Modelle basieren, wie z. B. dem Technology-, Pedagogy- and Computer-Knowledge-Modell (TPACK), das pädagogisches Wissen, Technikwissen und Fachwissen im Rahmen der Lernumgebung miteinander verknüpft (vgl. Mishra und Koehler 2006). Dennoch darf die Medienkompetenz der Studierenden nicht überschätzt werden. Die heutzutage alltägliche Nutzung von Social Media im privaten Bereich bringt nicht automatisch Fähigkeiten zum selbstregulierten Lernen und kritischen Umgang mit Medien mit sich, die an Hochschulen nötig sind. Nach Eschenauer entfalten sich die Dimensionen der Medienkompetenz in Auswahl und Nutzung von Medien zur Unterhaltung, Information, zum Spielen, Lernen, Problemlösen und Entscheidungen fällen, doch ebenso darin, Medienbotschaften zu bewerten und zu verstehen, sowie darstellende Medien angemessen zu gebrauchen (Eschenauer 1996, 32). Die Anforderungen an Studierende sind verstärkt erforschender und eigenständiger Natur (Aufgabenformate sowie Lernwege); wissenschaftliches Arbeiten wird immer mehr zum informellen sozialen Prozess, in dem der aktive Wissensaustausch in Gruppen und Netzwerken an Bedeutung gewinnt (vgl. Mürner und Polexe 2014, 6) und der anfangs angeleitete Lernprozess stärker in das Privatleben mit hineingenommen wird. Gerade in der Anfangszeit war eine intensive Betreuung der Gruppen 
nötig. Ein Wechsel von selbständigen Gruppenarbeitsphasen und Austauschphasen im Plenum, in denen die Gruppenfortschritte dokumentiert und beratschlagt wurden, erwies sich als effektiv. Wie bei einem Gerüst sollte den Studierenden so viel Hilfestellung angeboten werden, wie sie individuell benötigen, um schrittweise selbständiger zu werden, ohne andere durch zu viel Anleitung in ihrem Lernprozess einzuengen. Während der Veranstaltung wurde deutlich, dass leistungsschwache Gruppen mit der Offenheit der Aufgabenstellung eher schlecht zurechtkamen, was den Eindruck bestätigte, dass leistungsstarke Lernende von selbstbestimmten und offenen Aufgabenformaten am meisten profitierten, wenngleich das in einem Abschlusstest abgefragte Learning-Outcome aller Teilnehmenden als insgesamt hoch eingestuft werden konnte. ${ }^{2}$ Der fachliche Lernzuwachs war insgesamt auf hohem Niveau, die Gestaltung des Lernprozesses fiel den Gruppen unterschiedlich schwer oder leicht, was sich auch in der Qualität der Filme zeigte, wobei der Schwerpunkt explizit auf dem Lernprozess und nicht auf dem Endprodukt lag.

Die Identifikation mit dem Lerngegenstand war das markanteste Merkmal der Veranstaltung. Die Inhalte der Studierenden-erstellten Videos waren nach individuellen Interessen der Studierenden gewählt. Der persönliche Bezug zum Lerngegenstand, der im Allgemeinen nie rein rational, sondern auch emotional zu begründen ist (de Haan und Rülcker 2009, 181), ist dabei nicht zu unterschätzen. Denn aus konstruktivistischer Sicht stärken «[d] ie emotionale Identifikation mit dem Lerngegenstand, die Integration des neuen Wissens in das schon Bekannte, sowie zu wissen, dass man mit den neu erworbenen Fähigkeiten in antizipierten Handlungssituationen erfolgreich sein kann", die Bereitschaft, sich in Lernprozessen zu engagieren (ebd.). Bereits zu Beginn der Veranstaltung (Theorieblock) war die Motivation hoch, sich neues Wissen anzueignen, um die spätere Aufgabe bewältigen zu können und eigene Interessenschwerpunkte im Themenfeld auszumachen. Die Emotionalisierung mit dem selbstgewählten Lerngegenstand motivierte die Studierenden in der Filmphase, sich aktiv in ihr Projekt und das Seminar einzubringen sowie ein hohes Mass an Eigeninitiative aufzubringen. So berichteten mehrere Gruppen von freiwilliger Mehrarbeit (auch am Wochenende), eine Gruppe organisierte selbstständig eine gesamte Seminarsitzung, bei der sie einen renommierten Religionspädagogen interviewten. Zur Abschlussveranstaltung wurden Gäste eingeladen, um die Videos gemeinsam anzusehen und Feedback zu geben. Hier wurden weitere Dimensionen der Medienkompetenz nach Eschenauer $(1996,32)$ angebahnt: die Analyse von Medien und ihrer Produkte unter den spezifischen Rahmenbedingungen der Veranstaltung. Auch die Wahrnehmung der Medien in Bezug auf Emotionen, Vorstellungen und Verhaltensmustern wurde gestärkt. Dies wurde besonders deutlich bei einem energisch diskutierten Endergebnis, bei dem die Gruppe Menschen auf dem Campus zu ihren Vorurteilen gegenüber

2 Die Studierenden selbst meldeten einen hohen Lernzuwachs im religionspädagogischen Bereich zurück, der in einigen Videos, aber auch einem Abschlusstest, in hohem Masse bestätigt wurde. 
Lehramtsstudierenden mit dem Fach Religion befragten. Eine hohe Aktivierung der Studierenden wurde gewährleistet durch die Identifizierung mit dem Lerngegenstand und freie Wahlmöglichkeiten im Lernprozess. Das Medium wurde Mittel zum Zweck, anstatt zum «reinen Konsumationsmedium» (vgl. Wachtler und Ebner 2014). ${ }^{3}$ Alle Teilnehmenden meldeten einen hohen fachdidaktischen Lernzuwachs, aber auch einen Lernzuwachs im kritischen Umgang mit Medien zurück. Die grösste Herausforderung bildete für die Studierenden der rechtliche Rahmen im Umgang mit Medien. Dies wird auch in den Ergebnissen deutlich, da jedes Video urheberrechtliche Schwächen ${ }^{4}$ aufweist und die Ergebnisse somit der Öffentlichkeit nicht zugänglich gemacht werden können. Hier wäre eine Sitzung oder Selbstlernmaterial notwendig gewesen, um rechtliche Rahmenbedingungen zu klären.

\section{Fazit}

Die wesentlichen Erkenntnisse zeigen einen mediengestützten Mehrgewinn in den Bereichen Learning-Outcome, Motivation und Aktivierung im Lernprozess sowie Medienkompetenz besonders in Hinblick auf Analyse und Gestaltung von Lehrvideos auf. Als besonders spannend wurde die Arbeit mit der neuen Technik empfunden. Gerne wurden eigene Geräte eingesetzt, die auch im Privatleben genutzt werden. So diente beispielsweise das iPhone als Kamera und Bearbeitungsmedium, da es gleichzeitig geeignete Software (iMovie) bietet. Die neu gewonnenen Erkenntnisse in der Filmbearbeitung dienten neben der Verarbeitung religionspädagogischer Inhalte somit auch dem Privatleben (z. B. Erstellung von Videomaterial für Social Media Formate wie Facebook oder Instagram). Im Vergleich zu anderen Endprodukten (z. B. einzelne Kunstobjekte), sind Videos unvergänglich und konnten allen über das Internet oder Hardware unkompliziert zugänglich gemacht werden. Dies bietet vermutlich einen höheren Reiz, als beispielsweise ein Referat im Seminar abzuhalten, um den eigenen Lernprozess oder Lernzuwachs zu dokumentieren. Die eingangs formulierten Lernziele wurden erreicht. Der Wissenszuwachs sowie weiterführende Fragen deuten auf einen tiefen Lernprozess im Gegensatz zu trägem Wissen hin. Die hohe Aktivierung und Bereitschaft zu Mehrarbeit der Studierenden ist auf die (emotionale) Identifikation mit dem Lerngegenstand und freie Wahlmöglichkeiten, z. B. Mitbestimmen von Lerninhalten und Seminarablauf, zurückzuführen. Abschliessend kann davon ausgegangen werden, dass audiovisuelle Medien je nach didaktischer Einbettung und fachkompetenter Nutzung vielschichtige und tiefgreifende Lernprozesse

3 Videos sollten jedoch nicht mit herkömmlichen Methoden gleichgesetzt werden. Sie bilden lediglich ein einzelnes Stellrad in einem interdependenten Verhältnis von Ziel, Inhalt, Methoden und Medien (vgl. Meinhard et al. 2014, 52f.).

4 Das gilt etwa für die Untermalung des Videos mit Musik, ohne die Rechte dafür erworben zu haben oder auch dafür, Bildmaterial aus dem Internet zu nutzen und Personen zu filmen, von denen nicht sicher war inwiefern diese veröffentlicht werden dürfen. 
anregen können. In der beschriebenen Veranstaltung waren das gewählte Medium, Methoden und Aufgabenstellungen in einem durchdachten Lernszenario und einer transparenten Lehrendenrolle aufeinander abgestimmt, was den Erfolg ausmachte und den Mehrgewinn, angebunden an die spezifische Situation, erklärt.

\section{Literatur}

Eschenauer, Barbara. 1996. "Konferenzbericht» In Die Informationsgesellschaft von morgen Herausforderung an die Schule von heute, herausgegeben von Bertelsmann Stiftung, 32-32. Gütersloh: Bertelsmann Stiftung.

de Haan, Gerhard, und Tobias Rülcker. 2009. Der Konstruktivismus als Grundlage für die Pädagogik. Frankfurt am Main: Lang.

Kerres, Michael. 2013. Mediendidaktik. Konzeption und Entwicklung mediengestützter Lernangebote. 4., überarb. und aktualisierte Aufl. München: Oldenbourg.

Kleimann, Bernd, und Klaus Wannemacher. 2004. E-Learning an deutschen Hochschulen. Von der Projektentwicklung zur nachhaltigen Implementierung. Hannover: HIS GmbH.

Meinhard, David B., Ute Clemens, und Tobias Koch. 2014. "Zwischen Trend und Didaktik. Videos in der Hochschullehre.» Zeitschrift für Hochschulentwicklung 9 (3): 50-64. doi:10.3217/ zfhe-9-03/07.

Mürner, Beat, und Laura Polexe. 2014. «Digitale Medien im Wandel der Bildungskultur - neues Lernen als Chance.» Zeitschrift für Hochschulentwicklung 9 (3): 1-12. doi:10.3217/zfhe-9$03 / 02$.

de Witt, Claudia, und Michael Kerres. 2002. "Quo vadis Mediendidaktik? Zur theoretischen Fundierung von Mediendidaktik.» MedienPädagogik 6: 1-22. doi:10.21240/ mpaed/06/2002.11.08.X.

Mishra, Punya, und Matthew Koehler. 2006. «Technological pedagogical content knowledge: A framework for teacher knowledge.» Teachers College Record 108 (6): 1017-54. doi: 10.1111/ j.1467-9620.2006.00684.

Wachtler, Josef, und Martin Ebner. 2014. «Unterstützung von videobasiertem Unterricht durch Interaktionen - Implementierung eines ersten Prototyps.» Zeitschrift für Hochschulentwicklung 9 (3): 13-22. doi:10.3217/zfhe-9-03/03. 